TITLE:

\title{
Prefrontal cortex and neural mechanisms of executive function.
}

$\operatorname{AUTHOR}(S)$ :

Funahashi, Shintaro; Andreau, Jorge Mario

CITATION:

Funahashi, Shintaro ...[et al]. Prefrontal cortex and neural mechanisms of executive function.. Journal of physiology, Paris 2013, 107(6): 471-482

ISSUE DATE:

2013-12

URL:

http://hdl.handle.net/2433/179793

RIGHT:

(c) 2013 Published by Elsevier Ltd.; この論文は出版社版でありません。 引用の際には出版社版をご確認ご利用ください。; This is not the published version. Please cite only the published version. 


\title{
Prefrontal cortex and neural mechanisms of executive function
}

\author{
Shintaro Funahashi ${ }^{1,2)}$ and Jorge Mario Andreau ${ }^{2)}$
}

1) Kokoro Research Center, Kyoto University

Sakyo-ku, Kyoto 606-8501, Japan.

2) Department of Cognitive and Behavioral Sciences,

Graduate School of Human and Environmental Studies, Kyoto University

Sakyo-ku, Kyoto 606-8501, Japan.

Corresponding author:

Shintaro Funahashi

Kokoro Research Center, Kyoto University

46 Yoshida-Shimoadachi, Sakyo-ku, Kyoto 606-8501, Japan.

Phone \& Fax: (+81) 75-753-9674

E-mail: funahashi.shintaro.2z@kyoto-u.ac.jp 


\section{Abstract}

Executive function is a product of the coordinated operation of multiple neural systems and an essential prerequisite for a variety of cognitive functions. The prefrontal cortex is known to be a key structure for the performance of executive functions. To accomplish the coordinated operations of multiple neural systems, the prefrontal cortex must monitor the activities in other cortical and subcortical structures and control and supervise their operations by sending command signals, which is called top-down signaling. Although neurophysiological and neuroimaging studies have provided evidence that the prefrontal cortex sends top-down signals to the posterior cortices to control information processing, the neural correlate of these top-down signals is not yet known. Through use of the paired association task, it has been demonstrated that top-down signals are used to retrieve specific information stored in long-term memory. Therefore, we used a paired association task to examine the neural correlates of top-down signals in the prefrontal cortex. The preliminary results indicate that $32 \%$ of visual neurons exhibit pair-selectivity, which is similar to the characteristics of pair-coding activities in temporal neurons. The latency of visual responses in prefrontal neurons was longer than bottom-up signals but faster than top-down signals in inferior temporal neurons. These results suggest that pair-selective visual responses may be top-down signals that the prefrontal cortex provides to the temporal cortex, although further studies are needed to elucidate the neural correlates of top-down signals and their characteristics to understand the neural mechanism of executive control by the prefrontal cortex.

Keywords: Top-down signal, Working memory, Pair selectivity, Paired associate, Monkey 


\section{Introduction}

We often face various kinds of problems during our daily life. When we have a problem, we often need to make decisions quickly. Appropriate reasoning, judgment, and decision-making are essential if we wish to solve these problems promptly. However, reasoning, judgment, and decision-making are complex cognitive operations. Any of these functions needs to operate multiple neural systems simultaneously and in a coordinated manner. To operate multiple neural systems efficiently and to accomplish a specific goal successfully, some system in the brain needs to integrate and coordinate their operations. The mechanism that integrates and coordinates the operations of a variety of neural systems has been called "executive control" (Roberts, 1998; Miyake and Shah, 1999; Shah and Miyake, 1999). Executive function is considered to be a product of the coordinated operation of various neural systems and is essential for achieving a particular goal in a flexible and appropriate manner.

The prefrontal cortex has been demonstrated to be an important structure for executive functions (Baddeley, 1986; Stuss and Benson, 1986; Kolb and Whishaw, 1996; Fuster, 2008). Neuropsychological studies showed unique characteristics in prefrontal patients' behavior (Stuss and Benson, 1986). Patients with prefrontal damage usually show normal IQs in most psychological tests, have normal long-term memory functions, and exhibit normal perceptual, motor, and language skills. However, when these patients need to create a new and adaptive action program or choose the best among several equally probable alternatives, it becomes clear that their intellectual activity is profoundly disturbed. This disturbance of intellectual activity is caused by poor judgment, planning, and decision-making, and also by the poor temporal organization of behavior and poor working memory. Stuss and Benson (1986) summarized that frontal patients exhibit a lack of insight and foresight, diminished capacity for planning, and decreased initiative. Similar impairments have also been reported in animals with prefrontal lesions (see Fuster, 2008). These impairments 
cannot be explained simply by the failure of a particular function such as perception, motor, or long-term memory, but rather by a failure to coordinate the operation of perceptual, motor, and memory systems. The coordinated operation of perceptual, motor, memory, and other cognitive systems is essential for performing cognitive functions such as anticipation, planning, monitoring, and decision-making. Since these cognitive functions are included in executive functions, syndromes caused by prefrontal damage are often called "dysexecutive syndromes" (Stuss and Benson, 1986).

Thus, executive function can be explained as a product of the coordinated operation of various neural systems and is essential for any cognitive functions. Since the prefrontal cortex contributes to higher cognitive functions such as anticipation, judgment, planning, and decision-making, the prefrontal cortex is an important brain structure for performing executive functions. However, the neural mechanisms in the prefrontal cortex that are responsible for executive function are not yet well understood. Therefore, it is important to understand the characteristics of executive functions, how executive functions operate, and the neuronal mechanisms of executive functions.

\section{Executive functions}

Although executive functions are closely related to the functions of the prefrontal cortex, executive functions and executive control have not been well defined. A variety of definitions have been proposed. For example, Perner and Lang (1999) defined executive functions as functions "responsible for higher-level action control that are necessary in particular for maintaining a mentally specified goal and for bringing it to fruition against distracting alternatives." Baddeley and Della Sala (1998) defined executive function as the coordinated operation of multiple control systems that operate simultaneously. In their model of working memory, they considered executive function to be the function of the central executive. In their model, if the central executive became defective, it would be difficult to control slave systems (e.g., the visuospatial 
sketchpad and the phonological loop) simultaneously and in a coordinated manner. Therefore, they proposed that the characteristics of executive functions could be examined using a dual-task paradigm, in which the subjects are required to perform two different tasks (e.g., a visuospatial task and a linguistic task) simultaneously. Shallice and Burgess (1991) listed 5 types of situations that require a supervisory executive system: (1) planning or decision-making, (2) error-correction or troubleshooting, (3) performing responses that have not been well-learned or responses that contain novel sequences of action, (4) judging whether something is dangerous or technically difficult, and (5) overcoming a strong habitual response or resisting temptation. Burgess (1997) listed the following 5 functions as examples of executive functions: problem-solving, planning, initiation of activity, cognitive estimation, and prospective memory. Pineda et al. (1998) proposed that executive functions included the following 5 processes: self-regulation, control of cognition, temporal organization of the response to immediate stimuli, planning behavior, and control of attention. Rabbitt (1997) described seven features of executive control. First, executive control is necessary to deal with novel tasks. Second, executive control extends beyond the current internal or external environment to restructure an interpretation of the past as well as to attempt active control of the future. Third, executive control is necessary to initiate new sequences of behavior and also to interrupt other ongoing sequences of responses. Fourth, executive control is necessary to prevent inappropriate responses. Fifth, executive control can achieve rapid switching from one task to another. Sixth, executive control is necessary to monitor performance to correct errors, to alter plans, or recognize new opportunities. Seventh, executive control enables attention to be sustained continuously over long periods. Finally, Smith and Jonides (1999) summarized five functions as executive functions: (1) focusing attention on relevant information or processes and inhibiting irrelevant distractors (attention and inhibition), (2) scheduling processes in complex tasks (task management), which requires the switching of focused attention between tasks, (3) planning a sequence of subtasks to accomplish a goal (planning), (4) updating 
and checking the contents of working memory to determine the next step in a sequential task (monitoring), and (5) coding representations in working memory for the time and place of appearance (coding).

Although the definitions of executive function are somewhat different from researchers to researchers, some common features are present. Common features of executive function include the control of attention (switching attention from one source to another or focusing attention on one source), the temporal organization of behavior, the planning or scheduling of complex tasks to accomplish a future goal, the capacity to access and manipulate information stored in long-term memory, and the monitoring of current internal and external states. Executive function is a general term for these functions. Each of these functions is composed of multiple sub-functions each of which is supported by different neural systems. Therefore, to achieve executive functions, some neural system is necessary to coordinate and supervise the operation of these multiple neural systems.

The prefrontal cortex has been shown to play essential roles in executive functions and is considered to be an important brain structure that controls and supervises multiple neural systems located in other cortical and subcortical areas. In order for the prefrontal cortex to supervise a variety of cognitive functions and perform executive control, the prefrontal cortex needs to continuously monitor the activities in other cortical and sub-cortical areas and, at the same time, send command signals to these structures to control their operations. Thus, both the monitoring and controlling of activities in other brain areas are important functional components for performing executive function. Command signals that are provided by the prefrontal cortex and control the activities of other brain areas have been called "top-down signals." Although the presence of top-down signals has been supported by many studies (see the following sections), the nature of top-down signals is not yet entirely clear. Therefore, identification of the neural correlates and characteristics of top-down signals in the 
prefrontal cortex is important for understanding how the prefrontal cortex performs executive function and the neural mechanisms of executive control.

\section{Top-down control and its contribution to prefrontal executive functions}

\subsection{Top-down functional interaction between the prefrontal cortex and other cortical and subcortical areas}

The top-down modulation of activity by the prefrontal cortex has been demonstrated in neurophysiological studies as well as human brain imaging studies. Since broad anatomical connections are present between the prefrontal cortex and other cortical and subcortical structures (see Fuster, 2008), the top-down modulation by the prefrontal cortex could be supported by functional interactions between the prefrontal cortex and other cortical and subcortical structures through these anatomical connections. For example, the presence of functional interactions between the prefrontal cortex and the posterior cortices has been shown and these functional interactions support the presence of the top-down modulation of the activities in the posterior cortices by the prefrontal cortex. This functional interaction has been demonstrated by the effect of cooling in either cortical area. For example, Fuster et al. (1985) showed that the suppression of either prefrontal activities or inferior temporal activities by cooling led to the modulation of both spontaneous and task-related discharges and the diminution of stimulus selectivity in neurons of either intact cortex. Since cooling of either cortical area produced impairment in a delayed matching-to-sample task, they concluded that mutual functional interactions are present between these two cortical areas and that these interactions are necessary to perform cognitive tasks, such as a delayed matching-to-sample task. Quintana et al. (1989) examined the effects of parietal cooling on prefrontal activities during delay task performance. They observed that bilateral parietal cooling produced significant changes in spontaneous and task-related firings of 
prefrontal neurons together with behavioral changes such as misreaching, slow and inaccurate eye movements, and a longer choice reaction time. However, they did not find any behavioral impairment of delay task performance, indicating that performance of the delay task requires intact function of the prefrontal cortex. Therefore, they concluded that bilateral parietal cooling affected only bottom-up sensory transmission to the prefrontal cortex, and top-down information transmission from the prefrontal cortex was maintained and is important for monkeys to perform cognitive tasks. Thus, these cooling studies clearly demonstrated the presence of functional interactions between the prefrontal cortex and the posterior cortices. These studies also showed that prefrontal activity modulates neural operations in the posterior cortices and that this modulation plays an important role to perform cognitive tasks.

Top-down functional modulations by the prefrontal cortex have also been demonstrated by transcranial magnetic stimulation (TMS) studies in humans. Lee and D'Esposito (2012) applied theta burst TMS to the prefrontal cortex while human participants performed working memory tasks and examined responses caused by TMS in the prefrontal cortex and the extrastriate cortex using fMRI. They found that TMS disrupted prefrontal functions and that this disruption of prefrontal functions decreased the selectivity of extrastriate responses to sample stimuli and decreased working memory performance. Thus, the functional states of the prefrontal cortex could affect the functional states of the posterior cortices through top-down pathways.

Top-down functional interactions between the prefrontal cortex and the parietal cortex have also been demonstrated by developmental studies. Hwang et al. (2010) used oculomotor behaviors that required inhibitory control (anti- and pro-saccade tasks) and examined developmental changes in effective connectivity between prefrontal and parietal regions using fMRI. They found significant correlations between improvements in inhibitory control in oculomotor behavior and the age-related enhancement of top-down effective connectivity between frontal and parietal regions. In addition, Johnstone et al. (2007) showed the importance of top-down regulation in the 
prefrontal-subcortical circuitry for major depression. Thus, as was shown by anatomical studies, extensive functional interactions are present between the prefrontal cortex and other cortical and subcortical structures. The prefrontal cortex apparently uses these anatomical pathways for functional interactions to control the activities of cortical and subcortical structures. These functional interactions between the prefrontal cortex and other cortical and subcortical structures must be a fundamental component for the top-down modulation by the prefrontal cortex.

\subsection{Top-down modulation by the prefrontal cortex in attention}

Neural mechanisms of top-down modulation by the prefrontal cortex have been examined using attention paradigms in monkeys and humans. Prefrontal participation in the control of attention has been demonstrated by neurophysiological studies using monkeys. Among prefrontal cortical areas, the frontal eye fields (FEF) are known to be a source of attention signals. Therefore, neurophysiological studies regarding the control of attention have been performed in the FEF. Moor and Fallah (2004) studied the effects of microstimulation applied to the FEF to examine whether or not saccade-related mechanisms of the FEF provide a source of spatial attention. In their task, monkeys were required to detect changes in the luminance of a target while ignoring a flashing distractor. They found that the monkeys could detect smaller changes in target luminance if the change in luminance was preceded by FEF stimulation, which had a current intensity that was below the level that evoked saccades. Thus, FEF stimulation could increase the sensitivity to the target change and produce transient improvements in covert spatial attention. Similarly, Wardak et al. (2006) reversibly inactivated the FEF using microinjections of muscimol while monkeys performed a covert visual search task. Inactivation of the FEF caused spatially selective deficits in the execution of visual search tasks, such that it altered the ability to detect a visual target among distractors. Thus, activation and inactivation of the FEF produce 
spatially selective improvement and deficit in the execution of covert attention tasks, respectively. Further, Moore and Armstrong (2003) showed that visual responses in extrastriate visual area V4 are enhanced by FEF microstimulation when the location of the visual receptive field of the V4 neuron matches the region of the visual field represented at the stimulation site of the FEF. An enhancement of visual responses in visual areas by FEF activation could be a neural mechanism for focusing attention to a specific stimulus in a particular visual field. Thompson et al. (2005) showed that the source of attention signals in the FEF is the enhanced activity of visually responsive neurons. They proposed that spatially selective activity of visually responsive neurons in the FEF corresponds to the "mental spotlight" of attention through the modulation of ongoing visual processing. Thus, the activity of the FEF modulates the activity of other cortical or subcortical structures via afferent projections (Ninomiya et al., 2012). As was shown by Thompson et al. (2005), the spatially selective visual response in the FEF could be a neural correlate of a top-down signal for controlling attention.

Although the FEF plays an important role in the top-down control of attention, neuropsychological studies show that the prefrontal cortex also participates in the top-down control of attention in monkeys. Rossi et al. (2007) made unilateral lesions in the primate prefrontal cortex in combination with transection of the corpus callosum and the anterior commissure and asked monkeys to discriminate the target from the distractor presented in either visual hemifield. Although the monkeys exhibited no impairment when the cue was constantly presented in the affected hemifield for many trials, they were severely impaired when the position of the cue was switched frequently across trials. They concluded that the prefrontal cortex plays an essential role in the top-down control of attention to allocate attention flexibly on the basis of task demand.

Neurophysiological studies have also shown prefrontal participation in the top-down control of attention. For example, Everling et al. (2002) used a focused attention task in which monkeys were required to monitor a stream of visual objects and wait for a target object to appear at an attended location. They found that prefrontal 
neurons discriminated between targets and non-targets. However, they also found that the discrimination between targets and non-targets by prefrontal activity was diminished when the same object was presented at unattended locations. Thus, the prefrontal cortex participate in the active filtering of stimuli that are presented at unattended locations by the top-down modulation of the activity in the brain areas that provide visual information to the prefrontal cortex. Buschman and Miller (2007) examined the activity of both prefrontal neurons and posterior parietal neurons while monkeys performed visual attention tasks. In these tasks, monkeys were required to find a visual target under two conditions: visual "pop-out" (bottom-up) condition and visual search (top-down) condition. They used colored bar stimuli with different orientations as the target and the distractors. In the visual pop-out condition, the target and the distractors were identical, but the distractors were different from the target in two dimensions (stimulus color and orientation), so that the target's salience automatically drew attention to the target. In the visual search condition, each distractor independently differed from the target. Since the target matched some of the distractors in either dimension, monkeys needed to use remembered information regarding the target. They examined the response timing of neurons exhibiting target location selectivity between two conditions and found that prefrontal activities reflected the target location first during top-down conditions, whereas parietal activities reflected it first during bottom-up conditions. Therefore, they concluded that top-down signals arose from the prefrontal cortex when subjects performed attention tasks under the top-down condition. Thus, the prefrontal cortex participates in the top-down control of visual attention by filtering information in the brain areas that provide visual information to the prefrontal cortex.

Prefrontal participation in the top-down control of attention has also been demonstrated by human brain imaging studies. For example, Hopfinger et al. (2000) used a cued spatial attention task, in which the subject was required to discriminate black and white checkerboards from those containing some gray checks at the cued 
location. Two checkerboards were presented at both sides of the central fixation and the location that the subject paid attention (the cue) was indicated by yellow and blue arrows presented at the center of the screen. Subjects were told which color arrow to attend for the session. They showed that attention-directing cue (colored arrow) selectively activates the superior frontal, inferior parietal, and temporal cortices. Since subjects needed to direct their attention to the particular color of the arrow throughout the session, they concluded that these structures are part of a network for voluntary top-down attention control. Egner et al. (2008) examined brain areas that represented spatial- and feature-based search information and neural mechanisms for integrating top-down spatial- and feature-based information during visual search performance using fMRI. In their task, subjects were shown a cue stimulus followed by a search array, which consisted of four diamond stimuli peripherally placed (a blue and a red one to either side of the central fixation). Subjects needed to locate a target diamond, which was missing wither its upper or lower corner, and indicate which corner was missing by button press. The cue stimulus informed subjects which side they needed to attend (spatial-based search information) and which color they paid attention (feature-based search information). They found that spatial- and feature-based cue information were represented additively in frontal and parietal regions during preparation for a visual search. They suggested that this anticipatory integration is related to the generation of a "top-down salience map" (a search template of primed target locations and features) in these regions. Bressler et al. (2008) used Granger causality effects measured by BOLD signals while subjects performed a visual spatial attention task and showed that the activities of the FEF and the intraparietal sulcus (IPS) area modulate the activity of occipital visual areas. They showed that top-down levels of Granger causality from the FEF and the IPS to occipital visual areas were significantly greater than bottom-up levels and cortex-wide levels. In addition, they showed that Granger causality was significantly greater from the FEF to the IPS than from the IPS to the FEF. These results indicate that the FEF and the IPS modulate the activity of the occipital visual 
areas and the FEF modulates the activity of the IPS in relation to visual attention. Thus, the prefrontal cortex including the FEF plays an essential role in selective visual attention and participates in the control of attention using top-down command signals.

\subsection{Top-down modulation by the prefrontal cortex in long-term memory processes}

Although working memory has been focused strong attention as an important function of the prefrontal cortex, it has been known that the prefrontal cortex also participates in long-term memory processes and that the top-down control signal provided by the prefrontal cortex plays an important role in long-term memory processes, especially in memory encoding and retrieving processes. The importance of the prefrontal cortex in long-term memory processes is supported by neuropsychological and neuroimaging studies (Nolde et al., 1998; Buckner et al., 1999; Blumenfeld and Ranganath, 2007). Patients with prefrontal cortical damage exhibit impairments in the strategic utilization of memory. Their deficits are observed in domains such as free recall, proactive interference, temporal-order memory, and source memory (Fuster 2008). Neuroimaging studies have revealed prefrontal participation in long-term memory processes and regional differences in prefrontal roles in these processes. For example, the ventrolateral prefrontal regions have been shown to participate in memory encoding (Brewer et al., 1998; Wagner et al., 1998), whereas the anterior prefrontal regions participate in memory retrieval (Buckner and Koutsaal, 1998; Cabeza and Nyberg, 2000). Interestingly, it has been shown that the degree of activity in the ventrolateral prefrontal regions during encoding predicts the probability of the successful recall of memorized materials (Rugg et al., 1996; Brewer et al., 1998; Wagner et al., 1998). Hemispheric differences in prefrontal contribution to long-term memory processes have also been reported. Rossi et al. (2001) examined how the prefrontal cortex participates in episodic memory by transient functional interference using repetitive TMS applied to either the left or right prefrontal cortex. They found that the right prefrontal cortex is important for 
retrieving memorized items, whereas the left prefrontal cortex is important for encoding items. Buckner and Petersen (1996) also summarized hemispheric differences in the prefrontal role in memory retrieval. Both PET and fMRI studies showed that the left inferior prefrontal area was active during a wide range of tasks that required subjects to retrieve words or information about words from semantic memory, whereas the right anterior and posterior prefrontal areas were active during tasks that required subjects to retrieve information from specific episodes (episodic memory).

Several neuroimaging studies have shown interactions between working memory and long-term memory in the prefrontal cortex. For example, Braver et al. (2001) used the n-back task as a working memory task and the item encoding and retrieval tasks as long-term memory tasks, and examined regional differences in the participation of the prefrontal cortex in working and long-term memory tasks. They found that the dorsolateral prefrontal cortex mainly participates in working memory processes, whereas the ventrolateral prefrontal cortex participates in both encoding and retrieving processes of long-term memory. They also found that the anterior prefrontal region participates in both working and long-term memory tasks. On the other hand, Blumenfeld and Ranganath (2006) used two working memory conditions (rehearse trials and reorder trials) that required the subjects to retain triplets of words during a delay and examined prefrontal activity using fMRI. Behavioral analysis showed that the reorder condition enhanced long-term memory by strengthening inter-item association. fMRI analysis showed that dorsolateral prefrontal activity during reorder conditions was predictive of subsequent long-term memory performance. Therefore, they concluded that the dorsolateral prefrontal cortex contributes to long-term memory formation by strengthening associations among items that are organized in working memory (Blumenfeld and Ranganath, 2006, 2007).

Although the prefrontal cortex participates in long-term memory processes, functional connectivity between the prefrontal cortex and posterior cortices, especially the inferior temporal cortex, plays an important role in these processes (Buckner et al., 
1999; Simons and Spiers, 2003; Miyashita, 2004). This notion is supported by strong reciprocal anatomical connections between the prefrontal cortex and the temporal cortex (Petrides and Pandya, 1999, 2001). Recently, Ninomiya et al. (2012) used retrograde trans-synaptic tracing methods with rabies virus and double injections of two different dyes into areas MT and V4 and showed segregated projections from the prefrontal cortex to areas MT and V4 in macaque monkeys, such that area V4 primarily receives inputs from prefrontal area 46, while area MT receives inputs from both area 46 and the FEF through distinct FEF and parietal neurons. These results suggest that these segregated pathways from the frontal cortex carry functionally different top-down signals to different temporal areas.

In addition to anatomical studies, neurophysiological studies have also shown top-down influence by the prefrontal cortex in long-term memory processes. Hasegawa et al. (1998) used posterior-split-brain monkeys and showed that the retrieval of specific information stored in the temporal cortex as long-term memory is under the executive control of the prefrontal cortex. They used a visual paired association task, which requires the memory of stimulus-stimulus association. Stimulus-stimulus association acquired by the paired association task and stored in the temporal cortex does not usually transfer via the anterior corpus callosum. However, when a visual cue was introduced to one hemisphere in these split-brain monkeys, the anterior callosum instructed the other hemisphere to retrieve information regarding the paired associate of the visual cue. Since the anterior callosum is an important region for bilateral communication of the prefrontal cortex and since posterior-split-brain monkeys exhibited correct performances when a visual cue was presented at one visual field, they concluded that the retrieval of specific information stored in long-term memory is under the executive control of the prefrontal cortex. Tomita et al. (1999) also used posterior-split-brain monkeys and showed that a large number of inferior temporal neurons received top-down signals from the prefrontal cortex when bottom-up signals from the visual cortex were absent, while monkeys performed a paired association task. 
Behavioral performance was also impaired when top-down signals were not provided, which supports the importance of the top-down signal from the prefrontal cortex in paired association performance.

Thus, the prefrontal cortex plays a significant role in long-term memory processes through functional interactions with posterior cortices, especially the temporal cortex. Although the temporal cortex would be a storehouse of information for long-term memory, it has been shown that the prefrontal cortex provides top-down signals to correctly retrieve specific information stored in the temporal cortex.

\section{Importance of top-down signals to understand working memory and prefrontal functions}

The roles of top-down signals are important for understanding not only the neural mechanisms of executive functions but also the neural mechanisms of working memory. Working memory is an important function of the prefrontal cortex (Goldman-Rakic, 1987; Fuster, 2008). Working memory participates in a variety of higher cognitive functions such as reasoning, judgment, thinking, decision-making, and language comprehension, most of which are considered executive functions (Baddeley, 1986, Miyake and Shah, 1999). Working memory is described as a system that includes neural mechanisms for temporarily maintaining, manipulating and processing information in order to achieve a specific goal. Therefore, working memory needs to acquire specific information for the goal from a variety of sources and temporarily maintain it. At the same time, working memory provides maintained information to the neural system to achieve the goal. Thus, the neural mechanisms of working memory are also essential neural components for executive functions and could include similar neural processes as executive function could have, such as monitoring and controlling processes. Since working memory is an important function of the prefrontal cortex and since some neural mechanisms related to working memory have been proposed, we may be able to 
understand the neural mechanisms of executive function based on the mechanisms of working memory in the prefrontal cortex.

\subsection{Neural correlates of working memory processes in the prefrontal cortex}

The importance of working memory in understanding prefrontal functions has been supported by a variety of experiments, including lesion studies (see reviews by Goldman-Rakic, 1987; Petrides, 1994; Fuster, 2008), neurophysiological studies using non-human primates (see reviews by Funahashi and Kubota, 1994; Goldman-Rakic, 1998; Funahashi and Takeda, 2002; Fuster 2008), and neuroimaging studies using human subjects (see Stuss and Knight, 2002). Especially, neurophysiological investigations in the prefrontal cortex using working memory tasks have provided important data for understanding neural mechanisms that support working memory. For example, many prefrontal neurons exhibit tonic sustained activation (delay-period activity) during the delay period while monkeys perform spatial working memory tasks (Joseph and Barone, 1987; Funahashi et al., 1989; Sawaguchi and Goldman-Rakic, 1994; Hasegawa et al., 1998). This delay-period activity exhibited spatial selectivity, such that delay-period activity was observed only when the visual cue was presented at a particular location in the visual field. Delay-period activity was observed only when the subject performed correct responses. In addition, the duration of delay-period activity was prolonged or shortened depending on the length of the delay period. Based on these observations, delay-period activity has been considered to be a neural correlate of the mechanism for the temporary active maintenance of information in working memory processes (Goldman-Rakic, 1987; Funahashi, 2001; Funahashi and Takeda, 2002; Fuster, 2008).

Further, it has been shown that delay-period activity represents either retrospective information (e.g., the location of visual cue presentation) or prospective information (e.g., the direction of the forthcoming movement), although more prefrontal 
neurons encode retrospective information in delay-period activity (Funahashi et al., 1993; Takeda and Funahashi, 2002). A population vector analysis using a population of prefrontal activities revealed the alteration of the information represented by a population of prefrontal activities during the delay period, such that the direction of the population vector rotated gradually from the direction toward the visual cue to the direction toward the movement (Takeda and Funahashi, 2004). A cross-correlation analysis using simultaneously recorded pairs of prefrontal activities revealed functional interactions among a variety of task-related prefrontal neurons (Funahashi and Inoue, 2000; Constantinidis et al., 2001). Functional interactions were observed between neurons that exhibited different task-related activities or different spatial selectivities. The strength of these functional interactions changed depending on the task conditions. Temporal changes in functional interaction were also observed as the delay period progressed (Funahashi, 2001; Tsujimoto et al. 2008). Thus, functional interactions among prefrontal neurons with a variety of task-related activities and temporal as well as conditional changes in these interactions could play essential roles in processing and manipulating the information represented by a population of prefrontal neurons. A variety of functional interactions among prefrontal neurons must be a fundamental mechanism that underlies information processes in working memory.

\subsection{Models of working memory}

Several models of working memory have been proposed (see Miyake and Shah, 1999). The most influential model of working memory is that proposed by Baddeley (1986, 2000). His model of working memory includes a master system (the central executive) and three slave systems (the visuospatial sketchpad, the phonological loop, and the episodic buffer). Among these three slave systems, the phonological loop is a system for speech perception and language comprehension and includes mechanisms for temporarily maintaining speech-based information by sub-vocal rehearsal. The 
visuospatial sketchpad is a system for processing visuospatial information as well as information that cannot be processed by language. The episodic buffer is a temporary storage buffer with a limited capacity to integrate information that arises from a variety of sources including long-term memory. On the other hand, the central executive supervises the operation of these slave systems to achieve an appropriate goal. The central executive manages a limited capacity of memory resource and divides and re-organizes this memory resource depending on the demand from each slave system so that each slave system can accomplish the most effective operation under the current condition. Further, the central executive selects appropriate control processes or strategies for performing a current task accurately. Thus, the central executive supervises the operation of the slave systems by monitoring and controlling the operations of these systems. Although the central executive is considered to be the control mechanism for executive function, it is not clear how the central executive monitors and controls other systems.

The model of working memory proposed by Baddeley (2000) is an abstract model. No particular brain region or neural system is assigned to each of the four systems of working memory. However, working memory must be supported by certain and distinct neural systems in the brain. The prefrontal cortex has been considered to play a role as the central executive (Miyake and Shah, 1999). Working memory is defined as a system that includes neural mechanisms for temporarily maintaining, as well as manipulating and processing information. Neural mechanisms for temporarily maintaining and processing information are basic and essential mechanisms of any system for cognitive functions and, therefore, must be ubiquitous in the brain. These mechanisms must also be basic neural components, even in the central executive and three slave systems. Therefore, instead of an abstract model, we should construct a physiologically plausible model of working memory that includes neural mechanisms for temporarily maintaining and processing information. A model constructed with physiologically identifiable neural components would be useful for understanding the 
basic operations of working memory processes as well as how the prefrontal cortex participates in working memory and what prefrontal neural mechanism underlies working memory processes.

As shown in Fig. 1, we have proposed a model of working memory based on our findings obtained by neurophysiological studies in the prefrontal cortex (Funahashi, 2001). Since working memory is defined as a system that includes both the temporary maintenance of and processing of information, we hypothesized the presence of four basic neural processes for executing working memory. These include a process to select appropriate information (selection process), a process to temporarily store this information (temporary storage process), a process to provide information to other neural systems (output process), and a process to appropriately process information (processing process). In addition to the process for temporarily storing information and the process for processing information, we include two additional processes in our model of working memory. The process for temporarily storing information can receive various types of information, including sensory, motor, motivational, emotional, cognitive, and perhaps somatic information. However, the information that is temporarily maintained is that which is necessary for the current task. Therefore, a working memory model must include a process to select necessary information from a variety of sources. On the other hand, stored and processed information should be used for the task. For this purpose, the working memory model must have a process to provide information to other systems. Thus, when we consider a physiologically plausible model of working memory, the model should include at least the four neural processes shown in Fig. 1.

When we consider the neural correlates of these four processes in prefrontal activities, spatially selective delay-period activity could be a neural correlate of the process for temporarily storing information. Similarly, sensory responses such as cue-period activities and motor responses such as saccade-related activities could be parts of neural correlates related to the selection process and the output process, 
respectively. The input process receives necessary information from other brain areas and, at the same time, acts as a mechanism to monitor the operation of other brain areas. The output process sends information to be used by other brain areas or to control their operation. Target areas of the output process could be areas related to motor performance or long-term memory or areas for which the prefrontal cortex needs to control the activity to coordinate operations. Top-down control signals provided from the output process must play important roles to achieve the coordinated operation of multiple neural systems. Therefore, although the motor responses observed for prefrontal neurons are neural correlates of the output process, other types of activities including delay-period activity must also be considered neural correlates of the output process, especially when we consider these activities as neural correlates of top-down control signals.

On the other hand, in contrast to the input process, the output process, and the temporary storage process, the processing process cannot be considered to be the activity of a single neuron, but rather can be considered to be a product of the functional interactions among neurons that exhibit a variety of activities. Prefrontal neurons that exhibit delay-period activity have functional interactions with neurons that exhibit delay-period activity with a different spatial selectivity or neurons that exhibit other types of task-related activity (e.g., cue-period activity or saccade-related activity) (Funahashi and Inoue, 2000; Constantinidis et al. 2001). Dynamic and flexible modulation of the strength of functional interactions between neurons has been observed in the prefrontal cortex depending on the context of the behavioral task (Vaadia et al., 1995; Funahashi, 2001; Tsujimoto et al. 2008). Thus, these interactions and their dynamic modulation could be fundamental mechanisms for information processing. In addition to functional interactions among neurons, feed-back signals from motor centers (e.g., post-saccadic activity (Funahashi et al., 1991)), motivational or emotional information from the limbic areas (Barbas, 1992), and modulatory signals by catecholaminergic or monoaminergic inputs (Sawaguchi and Goldman-Rakic, 1994; 
Williams and Goldman-Rakic, 1995; Arnsten, 1998; Sawaguchi, 1998; Wang et al., 2004) could also act as signals to modulate the activity of the temporary storage process. Thus, information processing in working memory can be explained by dynamic interactions among neurons and groups of neurons and by the effects of modulatory chemical inputs on their activity.

\subsection{General-purpose and modality-specific working memory systems}

We proposed a physiologically plausible model of working memory based on neurophysiological data obtained from the prefrontal cortex while monkeys performed spatial working memory tasks. The unique feature of this working memory system operating in the prefrontal cortex is that it is not used for processing information with a specific purpose or a specific modality, such as only for processing visual information, controlling limb movements, or language comprehension. Since the prefrontal cortex has strong anatomical connections, mostly reciprocal connections, with the posterior association cortices (Petrides and Pandya, 1999, 2001; Fuster 2008), it can receive a variety of information including sensory, motor, motivational, or emotional information from other cortical and subcortical structures. In addition, the prefrontal cortex is located at the best anatomical position to monitor the functional state of the posterior cortices and to control their operation by sending top-down control signals through reciprocal corticocortical connections with posterior cortices. Therefore, the working memory system in the prefrontal cortex is used for information processing in general and is an essential neural component for the performance of any cognitive function in which the prefrontal cortex participates. Thus, the working memory system in the prefrontal cortex can act as a general-purpose information processing system that is commonly used to perform a variety of cognitive functions (see Fig. 2).

A system similar to the working memory system we proposed may also be necessary in the brain areas where sensory and motor information are processed. The 
working memory system that we proposed must also be present in the cortical areas where modality-specific information, such as sensory, motor, or emotional information, is processed, since the temporary storage of information and the processing of information are ubiquitous processes that are needed for any kind of information processing in the brain. In fact, tonic sustained delay-period activity has been observed not only in the prefrontal cortex, but also in the FEF (Funahashi et al., 1989; Lawrence et al., 2005; Roesch and Olson, 2005), the inferior temporal cortex (Fuster and Jervey, 1982; Miyashita and Chang, 1988; Fuster, 1990; Miller et al., 1993; Naya et al., 1996), the posterior parietal cortex (Murata et al., 1996; Chafee and Goldman-Rakic, 1998; Pesaran et al., 2002; Zhang and Barash, 2004; Sereno and Amador, 2006), and the premotor cortex (Weinrich and Wise, 1982; Kurata and Wise, 1988; Crammond and Kalaska, 2000; Ohbayashi et al., 2003). Thus, cortical areas in which modality-specific information is processed also contain the working memory system we proposed as an essential and basic neural component for information processing. However, since this working memory system processes a specific kind of information such as visual, auditory, or oculomotor information, for example, the working memory system in cortical areas where modality-specific information is processed can be called a modality-specific working memory system (see Fig. 2).

\subsection{Interactions between modality-specific and general-purpose working memory systems}

As shown in Fig. 2, modality-specific working memory is a mechanism for temporarily storing and processing one domain of information, such as sensory, emotional, motivational, linguistic, or motor information. A modality-specific working memory system is mostly present in modality-specific cortical areas and subcortical areas. On the other hand, general-purpose working memory is present in the prefrontal cortex. This system is a mechanism not only to temporarily store and process a variety of 
information but also to monitor and control the activities of the brain areas that perform modality-specific working memory. To actively control and supervise the activities of modality-specific working memory systems, the general-purpose working memory system sends top-down control signals to modality-specific working memory systems. Thus, the top-down control signal is the output from general-purpose working memory in the prefrontal cortex and could play important roles in controlling a variety of cognitive activities.

The functional interactions between the general-purpose working memory system and modality-specific working memory systems and the functions of the general-purpose working memory system shown in Fig. 2 reflect computational architectures similar to those of the working memory model with a connectionist framework proposed by Cohen and others (Cohen and Servan-Schreiber, 1992; Cohen et al., 1996; Miller and Cohen, 2001). They hypothesized that the prefrontal cortex represents the context information, which corresponds to the goal representation in the computational architecture of the production system. Context information can be defined as the information necessary to mediate an appropriate behavioral response. Context information includes a set of task instructions, a specific prior stimulus, or the result of processing a sequence of prior stimuli (Cohen et al., 1996). To accomplish a particular goal, some brain region needs to maintain an internal representation of the goal, to suppress unnecessary behaviors, and to temporally coordinate series of behaviors. Cohen et al. (1996) considered that the prefrontal cortex is the cortical region where these functions are mediated. Using the model we proposed in Fig. 2, prefrontal executive functions that Cohen and others proposed can be explained by functional interactions between the general-purpose working memory system in the prefrontal cortex and modality-specific working memory systems in other cortical areas through top-down control signals.

\section{Search for top-down signals in the prefrontal cortex}


A top-down control signal is an important component for understanding how the prefrontal cortex performs executive control and how the prefrontal cortex supervises and controls operations in other cortical and subcortical areas. However, although there is evidence that the prefrontal cortex controls other cortical and subcortical activities by top-down signals, it is not known which activity is a neural correlate of the top-down signal and how this activity acts as a control signal.

The prefrontal cortex is known to play an important role in retrieving information from long-term memory. In addition, the prefrontal cortex has been shown to send top-down signals to posterior cortices during the retrieval of information from long-term memory. The function of the prefrontal top-down signal has been examined in the inferior temporal (IT) cortex. Sakai and Miyashita (1991) used a visual paired association task with 12 arbitrarily constructed pairs of fractal images and recorded single-neuron activities from the IT cortex. They found two kinds of task-related neurons in the IT cortex (pair-coding neurons and pair-recall neurons). Among these, pair-coding neurons represented information regarding a particular pair of pictures and exhibited the strongest and second-strongest responses during sample presentation when the pair of stimuli was presented as a sample stimulus. Subsequently, by using posterior-split-brain monkeys that performed the paired association task, Hasegawa et al. (1998) showed that, although the IT cortex participates in the storage of information as long-term memory, the retrieval of specific information from long-term memory is under the executive control of the prefrontal cortex (Miyashita and Hayashi, 2000). In addition, Tomita et al. (1999) showed that a large number of IT neurons received top-down signals from the prefrontal cortex as well as bottom-up signals from the visual cortex, and that the onset latency of sample-period activity under the top-down condition was significantly longer than that under the bottom-up condition. These results strongly support the notion that the prefrontal cortex plays an important role in retrieving specific information from long-term memory and indicate that the top-down 
signals generated in the prefrontal cortex play an essential role in memory-retrieval processes from long-term memory.

The paired association task requires the subject to remember an association between arbitrarily constructed paired symbols or images. During performance of the paired association task, subjects are required to retrieve specific information associated with the sample stimulus from long-term memory. Therefore, the paired association task is an appropriate task for examining the neural mechanisms of information retrieval from long-term memory in animals. Based on observations in the IT cortex and the advantages offered by using the paired association task, to identify neural correlates of top-down signals in the prefrontal cortex, we analyzed prefrontal single-neuron activities while monkeys performed a visual paired association task using 12 pairs of visual stimuli (Andreau and Funahashi, 2011).

We used a paired association task with a Go/No-Go response (Fig. 3). In this task, when monkeys pressed a lever, a fixation spot appeared at the center of the monitor. After a 1-s fixation period, the sample stimulus was presented on the monitor for a 0.5 -s sample period. The sample stimulus was randomly selected from 24 visual stimuli. At the end of the sample period, the fixation spot was presented again and a 5-s delay 1 period was introduced. The monkey was required to look at the fixation spot during the delay 1 period. At the end of the delay 1 period, another visual stimulus was presented on the monitor. The stimulus was either a paired associate of the sample stimulus (matching stimulus) or any of the remaining 22 stimuli (distractor stimulus). If the matching stimulus was presented, the monkey was required to release the lever within $0.5 \mathrm{~s}$ (Go condition) to receive a reward. If a distractor stimulus was presented, the monkey was required to continue to hold the lever until the end of the 1-s delay 2 period (No-Go condition) and release the lever within $0.5 \mathrm{~s}$ when the matching stimulus was presented.

We collected the activities of 217 neurons from the lateral prefrontal cortex. Among them, 68 showed statistically significant sample-period activity. A large number 
of neurons with sample-period activity exhibited selectivity to sample stimuli. The response magnitude to the presentation of the sample stimulus was different depending on which stimulus was presented as sample stimulus. Thus, neurons exhibited a variety of stimulus selectivity. Among these neurons with stimulus selectivity, some neurons exhibited significant excitatory responses when either stimulus of a pair was presented as sample stimulus. When the neurons exhibited the largest response to a particular sample stimulus and the second largest response to the paired associate of that stimulus, Sakai and Miyashita (1991) defined these neurons having pair selectivity in the IT cortex. Among prefrontal neurons exhibiting stimulus selectivity, 32\% showed similar characteristics as IT neurons having pair selectivity. Therefore, we classified these prefrontal neurons as having pair selectivity during the sample period (Fig. 4). Sakai and Miyashita (1991) calculated pair indices (PI) using sample-period activities to depict the strength of pair selectivity in IT neurons. To examine the strength of pair selectivity in prefrontal neurons and compare the strength of pair selectivity between prefrontal neurons and IT neurons, we calculates pair indices using same formulae proposed by Sakai and Miyashita (1991). We found that more neurons in the prefrontal cortex had positive PI values than those reported in the IT cortex, suggesting that the strength of pair selectivity is stronger in prefrontal neurons than IT neurons. This difference suggests that prefrontal neurons encode associative information of visual stimuli more strongly than IT neurons in paired association performance (Andreau and Funahashi, 2011).

As explained before, Tomita et al. (1999) showed that a large number of IT neurons received top-down signals from the prefrontal cortex as well as bottom-up signals from the visual cortex, and that the onset latency of sample-period activity under the top-down condition was significantly longer than that under the bottom-up condition. If the onset latency of sample-period activity observed in prefrontal neurons was significantly longer than that in IT neurons under the bottom-up condition and, at the same time, significantly shorter than that in IT neurons under the top-down condition, 
the results strongly support the notion that sample-period activity observed in the prefrontal cortex acts as the top-down signal to activate IT neurons and that the top-down signals generated in the prefrontal cortex play an essential role in memory-retrieval processes from long-term memory. In fact, the mean onset latency of sample-period activity in prefrontal neurons (144 ms) was longer than the latency (73 $\mathrm{ms}$ ) observed in IT neurons when visual information was provided through bottom-up pathways, but shorter than the latency $(178 \mathrm{~ms})$ observed in IT neurons when visual information was provided through top-down pathways (Tomita et al., 1999). These results suggest that prefrontal neurons play significant roles in retrieving associative information of visual stimuli and indicate that pair-selective sample-period activity might be a candidate of the top-down signal that the prefrontal cortex provides to the IT cortex for retrieving specific information assigned by the sample stimulus (Andreau and Funahashi, 2011).

\section{Conclusions}

Executive function is a product of the coordinated operation of various neural systems and is essential for achieving a particular goal in a flexible manner. The prefrontal cortex has been shown to be an important brain structure for executive control. To conduct coordinated operations to achieve a particular goal, the prefrontal cortex is thought to monitor other cortical and subcortical structures and control their operations by sending control signals called top-down signals. Although neurophysiological and neuroimaging studies have provided evidence that the prefrontal cortex sends top-down signals to control information processing in the posterior cortices, the neural correlate of the top-down signal is not yet known. An examination of the neural correlate of the top-down signal should provide important information for understanding the neural mechanism of executive control in the prefrontal cortex. Therefore, we tried to identify neural correlates of top-down signals in the prefrontal cortex. Top-down signals of the 
prefrontal cortex are used to retrieve specific information stored in long-term memory and play a role to produce "pair-recall" activity in IT neurons, which reflects the retrieval of a paired associate, while monkeys perform a paired association task (Sakai and Miyashita, 1991). Therefore, we used a paired association task with 12 pairs of visual stimuli to examine the neural correlates of top-down signals in the prefrontal cortex. Among neurons with a visual response, $32 \%$ showed pair-selectivity, similar to pair-coding activity in IT neurons. The latency of the visual responses of prefrontal neurons was longer than the bottom-up signals but faster than the top-down signals observed in IT neurons (Andreau and Funahashi, 2011). These results indicate that the prefrontal cortex participates in retrieving information from long-term-memory and that pair-selective visual responses could be a candidate for the neural correlate of the top-down signals that the prefrontal cortex provides to the IT cortex. 


\section{Acknowledgment}

This work was supported by Grants-in-Aid for Scientific Research from the Japanese Ministry of Education, Culture, Sports, Science and Technology (Nos. 17300103, 21240024, 22220003).

Current address of Jorge Mario Andreau: Unidad de Neurobiologia Aplicada (CEMIC-CONICET), Av. Galvan 4102, C1431FWO, Buenos Aires, Argentina. 


\section{Reference}

Andreau, J.M. and Funahashi, S., 2011. Primate prefrontal neurons encode the association of paired visual stimuli during the pair-association task. Brain Cogn. 76, $58-69$.

Arnsten, A.F.T., 1998. Catecholamine modulation of prefrontal cortical cognitive function. Trends Cogn. Sci. 2, 436-447.

Baddeley, A., 1986. Working Memory, Oxford University Press, Oxford.

Baddeley, A., 2000. The episodic buffer: a new component of working memory? Trends Cogn. Sci. 4, 417-423.

Baddeley, A. and Della Sala, D., 1998. Working memory and executive control. In: Roberts, A.C., Robbins, T.W., and Weiskrantz, L. (Eds.), The Prefrontal Cortex: Executive and Cognitive Functions. Oxford University Press, Oxford, pp. 9-21.

Barbas, H., 1992. Architecture and cortical connections of the prefrontal cortex in the rhesus monkey. In: A.V. Chauvel et al. (Eds.), Advances in Neurology. Vol. 57. Raven Press, New York, pp. 91-115.

Blumenfeld, R.S. and Ranganath, C., 2006. Dorsolateral prefrontal cortex promotes long-term memory formation through its role in working memory organization. J. Neurosci. 26, 916-925.

Blumenfeld, R.S. and Ranganath, C., 2007. Prefrontal cortex and long-term memory encoding: an integrative review of findings from neuropsychology and neuroimaging. Neuroscientist 13, 280-291.

Braver, T.S., Barch, D.M., Kelley, W.M., Buckner, R.L., Cohen, N.J., Miezin, F.M., Snyder, A.Z., Ollinger, J.M., Akbudak, E., Conturo, T.E., and Petersen, S.E., 2001. Direct comparison of prefrontal cortex regions engaged by working and long-term memory tasks. Neuroimage 14, 48-59. 
Bressler, S.L., Tang, W., Sylvester, C.M., Shulman, G.L., and Corbetta, M., 2008. Top-down control of human visual cortex by frontal and parietal cortex in anticipatory visual spatial attention. J. Neurosci. 28, 10056-10061.

Brewer, J.B., Zhao, Z., Glover, G.H., and Gabrieli, J.D.E., 1998. Making memories: brain activity that predicts how well visual experience will be remembered. Science $281,1185-1187$.

Buckner, R.L., Kelley, W.M., and Petersen, S.E., 1999. Frontal cortex contributes to human memory formation. Nature Neurosci. 2, 311-314.

Buckner, R.L. and Koutsaal, W., 1998. Functional neuroimaging studies of encoding, priming and explicit memory retrieval. Proc. Natl. Acad. Sci. USA. 95, 891-898.

Buckner, R.L. and Petersen, S.E., 1996. What does neuroimaging tell us about the role of prefrontal cortex in memory retrieval? Seminars Neurosci. 8, 47-55.

Burgess, P.W., 1997. Theory and methodology in executive function research. In: Rabbitt, P. (Ed.), Methodology of Frontal and Executive Function. Psychology Press, East Sussex, UK, pp. 81-116.

Buschman, T.J. and Miller, E.K., 2007. Top-down versus bottom-up control of attention in the prefrontal and posterior parietal cortices. Science. 315, 1860-1862.

Cabeza, R. and Nyberg, L., 2000. Imaging cognition II: an empirical review of 275 PET and fMRI studies. J. Cogn. Neurosci. 12, 1-47.

Chafee, M.V. and Goldman-Rakic, P.S., 1998. Matching patterns of activity in primate prefrontal area 8a and parietal area 7ip neurons during a spatial working memory task. J. Neurophysiol. 79, 2919-2940.

Cohen, J.D., Braver, T.S., and O'Reilly, R.C., 1996. A computational approach to prefrontal cortex, cognitive control and schizophrenia: recent developments and current challenges. Phil. Trans. R. Soc. London B 351, 1515-1527. 
Cohen, J.D. and Servan-Schreiber, D., 1992. Context, cortex, and dopamine: a connectionist approach to behavior and biology in schizophrenia. Psychol. Rev. 99, 45-77.

Constantinidis, C., Franowicz, M.N., and Goldman-Rakic, P.S., 2001. Coding specificity in cortical microcircuits: a multiple-electrode analysis of primate prefrontal cortex. J. Neurosci. 21, 3646-3655.

Crammond, D.J. and Kalaska, J.F., 2000. Prior information in motor and premotor cortex: activity during the delay period and effect on pre-movement activity. J. Neurophysiol. 84, 986-1005.

Egner, T., Monti, J.M.P., Trittschuh, E.H., Wieneke, C.A., Hirsch, J., and Mesulam, M.-M., 2008. Neural integration of top-down spatial and feature-based information in visual search. J. Neurosci. 28, 6141-6151.

Everling, S., Tinsley, C.J., Gaffan, D., and Duncan, J., 2002. Filtering of neural signals by focused attention in the monkey prefrontal cortex. Nature Neurosci. 5, 671-676.

Funahashi, S., 2001. Neuronal mechanisms of executive control by the prefrontal cortex. Neurosci. Res. 39, 147-165.

Funahashi, S., Bruce, C.J., and Goldman-Rakic, P.S., 1989. Mnemonic coding of visual space in the monkey's dorsolateral prefrontal cortex. J. Neurophysiol. 61, 331-349.

Funahashi, S., Bruce, C.J., and Goldman-Rakic, P.S., 1991. Neuronal activity related to saccadic eye movements in the monkey's dorsolateral prefrontal cortex. J. Neurophysiol. 65, 1464-1483.

Funahashi, S., Chafee, M.V., and Goldman-Rakic, P.S., 1993. Prefrontal neuronal activity in rhesus monkeys performing a delayed anti-saccade task. Nature 365, 753-756.

Funahashi, S. and Inoue, M., 2000. Neuronal interactions related to working memory processes in the primate prefrontal cortex revealed by cross-correlation analysis. Cereb. Cortex 10, 535-551. 
Funahashi, S. and Kubota, K., 1994. Working memory and prefrontal cortex. Neurosci. Res. 21, 1-11.

Funahashi, S. and Takeda, K., 2002. Information processes in the primate prefrontal cortex in relation to working memory processes. Rev. Neurosci. 13, 313-346.

Fuster, J.M., 1990. Inferotemporal units in selective visual attention and short-term memory. J. Neurophysiol. 64, 681-697.

Fuster, J.M., 2008. The Prefrontal Cortex. Fourth Edition, Elsevier, London, UK.

Fuster, J.M., Bauer, R.H., and Jervey, J.P., 1985. Functional interactions between inferotemporal and prefrontal cortex in a cognitive task. Brain Res. 330, 299-307.

Fuster, J.M. and Jervey, J.P., 1982. Neuronal firing in the inferotemporal cortex of the monkey in a visual memory task. J. Neurosci. 2, 361-375.

Goldman-Rakic, P.S., 1987. Circuitry of primate prefrontal cortex and regulation of behavior by representational memory. In: F. Plum (Ed.) Higher Functions of the Brain, Part 1, Handbook of Physiology, Section 1: The Nervous System, Vol. V, American Physiological Society, Bethesda, MD, pp. 373-417.

Goldman-Rakic, P.S., 1998. The prefrontal landscape: implications of functional architecture for understanding human mentation and the central executive. In: A.C. Roberts, T.W. Robbins, and L. Weiskrantz (Eds.), The Prefrontal Cortex: Executive and Cognitive Functions. Oxford University Press, Oxford, UK, pp. 87-102.

Hasegawa, I., Fukushima, T., Ihara, T., and Miyashita, Y., 1998. Callosal window between prefrontal cortices: cognitive interaction to retrieve long-term memory. Science 281, 814-818.

Hasegawa, R., Sawaguchi, T., and Kubota, K., 1998. Monkey prefrontal neuronal activity coding the forthcoming saccade in an oculomotor delayed matching-to-sample task. J. Neurophysiol. 79, 322-333.

Hopfinger, J.B., Buonocore, M.H., and Mangum, G.R., 2000. The neural mechanisms of top-down attentional control. Nature Neurosci. 3, 284-291. 
Hwang, K., Velanova, K., and Luna, B., 2010. Strengthening of top-down frontal cognitive control networks underlying the development of inhibitory control: a functional magnetic resonance imaging effective connectivity study. J. Neurosci. 30, 15535-15545.

Johnstone, T., van Reekum, C.M., Urry, H.L., Kalin, N.H., and Davidson, R.J., 2007. Failure to regulate: counterproductive recruitment of top-down prefrontal-subcortical circuitry in major depression. J. Neurosci. 27, 8877-8884.

Joseph, J.P. and Barone, P., 1987. Prefrontal unit activity during a delayed oculomotor task in the monkey. Exp. Brain Res. 67, 460-468.

Kolb, B. and Whishaw, I.Q., 1996. Fundamentals of Human Neuropsychology. Fourth Edition. W.H. Freeman, New York.

Kurata, K. and Wise, S.P., 1988. Premotor cortex of rhesus monkeys: set-related activity during two conditional motor tasks. Exp. Brain Res. 69, 327-343.

Lawrence, B.M., White, III, R.L., and Snyder, L.H., 2005. Delay-period activity in visual, visuomovement, and movement neurons in the frontal eye field. J. Neurophysiol. 94, 1498-1508.

Lee, T.G. and D'Esposito, M., 2012. The dynamic nature of top-down signals originating from prefrontal cortex: a combined fMRI-TMS study. J. Neurosci. 32, 15458-15466.

Miller, E.K. and Cohen, J.D., 2001. An integrative theory of prefrontal cortex function. Annu. Rev. Neurosci. 24, 167-202.

Miller, E.K., Lin, L., and Desimone, R., 1993. Activity of neurons in anterior inferior temporal cortex during a short-term memory task. J. Neurosci. 13, 1460-1478.

Miyake, A. and Shah, P., 1999. Models of Working Memory: Mechanisms of Active Maintenance and Executive Control. Cambridge University Press, Cambridge, UK.

Miyake, A. and Shah, P., 1999. Toward unified theories of working memory: emerging general consensus, unresolved theoretical issues, and future research directions. In: 
Miyake, A. and Shah, P. (Eds.), Models of Working Memory: Mechanisms of Active Maintenance and Executive Control. Cambridge University Press, Cambridge, UK, pp. $442-481$.

Miyashita, Y., 2004. Cognitive memory: cellular and network machineries and their top-down control. Science 306, 435-440.

Miyashita, Y. and Chang, H.S., 1988. Neuronal correlate of pictorial short-term memory in the primate temporal cortex. Nature 331, 68-70.

Miyashita, Y. and Hayashi, T., 2000. Neural representation of visual objects: encoding and top-down activation. Curr. Opinion Neurobiol. 10, 187-194.

Moore, T. and Armstrong, K.M., 2003. Selective gating of visual signals by microstimulation of frontal cortex. Nature 421, 370-373.

Moore, T. and Fallah, M., 2004. Microstimulation of the frontal eye field and its effects on covert spatial attention. J. Neurophysiol. 91, 152-162.

Murata, A., Gallese, V., Kaseda, M., and Sakata, H., 1996. Parietal neurons related to memory-guided hand manipulation. J. Neurophysiol. 75, 2180-2186.

Naya, Y., Sakai, K., and Miyashita, Y., 1996. Activity of primate inferotemporal neurons related to a sought target in pair-association task. Proc. Natl. Acad. Sci. USA 93, 2664-2669.

Ninomiya, T., Sawamura, H., Inoue, K.-I., and Takada, M., 2012. Segregated pathways carrying frontally derived top-down signals to visual areas MT and V4 in macaques. J. Neurosci. 32, 6851-6858.

Nolde, S.F., Johnson, M.K., and Raye, C.L., 1998. The role of prefrontal cortex during tests of episodic memory. Trends Cogn. Sci. 2, 399-406.

Ohbayashi, M., Ohki, K., and Miyashita, Y., 2003. Conversion of working memory to motor sequence in the monkey premotor cortex. Science 301, 233-236.

Perner, J. and Lang, B., 1999. Development of theory of mind and executive control. Trends Cogn. Sci. 3, 337-344. 
Pesaran, B., Pezaris, J.S., Sahani, M., Mitra, P.P., and Andersen, R.A., 2002. Temporal structure in neuronal activity during working memory in macaque parietal cortex. Nature Neurosci. 5, 805-811.

Petrides, M., 1994. Frontal lobes and working memory: evidence from investigations of the effects of cortical excisions in nonhuman primates. In: F. Boller, H. Spinnler, and J.A. Hendler (Eds.), Handbook of Neuropsychology, Vol. 9, Elsevier, Amsterdam, pp. 17-58.

Petrides, M. and Pandya, D.N., 1999. Dorsolateral prefrontal cortex: comparative cytoarchitectonic analysis in the human and the macaque brain and corticocortical connection patterns. Eur. J. Neurosci. 11, 1011-1036.

Petrides, M. and Pandya, D.N., 2001. Comparative cytoarchitectonic analysis of the human and the macaque ventrolateral prefrontal cortex and corticocortical connection patterns in the monkey. Eur. J. Neurosci. 16, 291-310.

Pineda, D., Ardila, A., Rosselli, M., Cadavid, C., Mancheno, S., and Mejia, S., 1998. Executive dysfunctions in children with attention deficit hyperactivity disorder. Int. J. Neurosci. 96,177-196.

Quintana, J., Fuster, J.M., and Yajeya, J., 1989. Effects of cooling parietal cortex on prefrontal units in delay tasks. Brain Res. 503, 100-110.

Rabbitt, P., 1997. Introduction: methodologies and models in the study of executive function. In: Rabbitt, P. (Ed.), Methodology of Frontal and Executive Function. Psychology Press, Hove, East Sussex, UK, pp. 1-38.

Roberts, A.C., 1998. Introduction. In: Roberts, A.C., Robbins, T.W., and Weiskrantz, L. (Eds.), The Prefrontal Cortex: Executive and Cognitive Functions. Oxford University Press, Oxford, UK, pp. 1-8.

Roesch, M.R. and Olson, C.R., 2005. Neuronal activity dependent on anticipated and elapsed delay in macaque prefrontal cortex, frontal and supplementary eye fields, and premotor cortex. J. Neurophysiol. 94, 1469-1497. 
Rossi, A.F., Bichot, N.P., Desimone, R., and Ungerleider, L.G., 2007. Top-down attentional deficits in macaques with lesions of lateral prefrontal cortex. J. Neurosci. 27, 11306-11314.

Rossi, S., Cappa, S.F., Babiloni, C., Pasqualetti, P., Miniussi, C., Carducci, F., Babiloni, F., and Rossini, P.M., 2001. Prefrontal cortex in long-term memory: an "interference" approach using magnetic stimulation. Nature Neurosci. 4, 948-952.

Rugg, M.D., Fletcher, P.C., Frith, C.D., Frackowiak, R.S.J., and Dolan, R.J., 1996. Differential activation of the prefrontal cortex in successful and unsuccessful memory retrieval. Brain 119, 2073-2083.

Sakai, K. and Miyashita, Y., 1991. Neural organization for the long-term memory of paired associates. Nature 354, 152-155.

Sawaguchi, T., 1998. Attenuation of delay-period activity of monkey prefrontal neurons by an alpha2-adrenergic antagonist during an oculomotor delayed-response task. J. Neurophysiol. 80, 2200-2205.

Sawaguchi, T. and Goldman-Rakic, P.S., 1994. The role of D1-dopamine receptor in working memory: local injections of dopamine antagonists into the prefrontal cortex of rhesus monkeys performing an oculomotor delayed-response task. J. Neurophysiol. 71, 515-528.

Sereno, A.B. and Amador, S.C., 2006. Attention and memory-related responses of neurons in the lateral intraparietal area during spatial and shape-delayed match-to-sample tasks. J. Neurophysiol. 95, 1078-1098.

Shah, P. and Miyake, A., 1999. Models of working memory: an introduction. In: Miyake, A. and Shah, P. (Eds.), Models of Working Memory: Mechanisms of Active Maintenance and Executive Control. Cambridge University Press, Cambridge, UK, pp. 1-27.

Shallice, T. and Burgess, P., 1991. Higher-order cognitive impairments and frontal lobe lesions in man. In: Levin, H.S., Eisenberg, H.M., and Benton, A.L. (Eds.) Frontal 
Lobe Function and Dysfunction. Oxford University Press, New York, USA, pp. $125-138$.

Simons, J.S. and Spiers, H.J., 2003. Prefrontal and medial temporal lobe interactions in long-term memory. Nature Rev. Neurosci. 4, 637-648.

Smith, E.E. and Jonides, J., 1999. Storage and executive processes in the frontal lobes. Science 283, 1657-1661.

Stuss, D.T. and Benson, D.F., 1986. The Frontal Lobes. Raven Press, New York.

Stuss, D.T. and Knight, R.T., 2002. Principles of Frontal Lobe Function, Oxford University Press, New York.

Takeda, K. and Funahashi, S., 2002. Prefrontal task-related activity representing visual cue location or saccade direction in spatial working memory tasks. J. Neurophysiol. $87,567-588$.

Takeda, K. and Funahashi, S., 2004. Population vector analysis of primate prefrontal activity during spatial working memory. Cereb. Cortex 14, 1328-1339.

Thompson, K.G., Biscoe, K.L., and Sato, T.R., 2005. Neuronal basis of covert spatial attention in the frontal eye field. J. Neurosci. 25, 9479-9487.

Tomita, H., Ohbayashi, M., Nakahara, K., Hasegawa, I., and Miyashita, Y., 1999. Top-down signal from prefrontal cortex in executive control of memory retrieval. Nature 401, 699-703.

Tsujimoto, S., Genovesio, A., and Wise, S.P., 2008. Transient neuronal correlations underlying foal selection and maintenance in prefrontal cortex. Cereb. Cortex 18, 2748-2761.

Vaadia, E., Haalman, I., Abeles, M., Bergman, H., Prut, Y., Slovin, H., and Aertsen, A., 1995. Dynamics of neuronal interactions in monkey cortex in relation to behavioral events. Nature 373, 515-518. 
Wagner, A.D., Schacter, D., Rotte, M., Koutstaal, W., Maril, A., Dale, A.M., Rosen, B.R., and Buckner, R.L., 1998. Building memories: Remembering and forgetting of verbal experiences as predicted by brain activity. Science $281,1188-1191$.

Wang, M., Vijayraghavan, S., and Goldman-Rakic, P.S., 2004. Selective D2 receptor actions on the functional circuitry of working memory. Science 303, 853-856.

Wardak, C., Ibos, G., Duhamel, J.-R., and Olivier, E., 2006. Contribution of the monkey frontal eye field to covert visual attention. J. Neurosci. 26, 4228-4235.

Weinrich, M. and Wise, S.P., 1982. The premotor cortex of the monkey. J. Neurosci. 2, $1329-1345$.

Williams, G.V. and Goldman-Rakic, P.S., 1995. Modulation of memory fields by dopamine D1 receptors in prefrontal cortex. Nature 376, 572-575.

Zhang, M. and Barash, S., 2004. Persistent LIP activity in memory antisaccades: working memory for a sensorimotor transformation. J. Neurophysiol. 91, 1424-1441. 


\section{Legends of Figures}

Fig. 1. A model for explaining a neural mechanism of working memory. This model is based on the results obtained by neurophysiological studies in the prefrontal cortex using spatial working memory tasks. (adapted from Funahashi, 2001).

Fig. 2. A schematic representation of functional interactions between a general-purpose working memory system and modality-specific working memory systems. Each working memory system includes neural components shown in Fig. 1, but processes different types of information.

Fig. 3. A. Schematic drawing of a paired association task that we used. B. Twelve pairs of visual stimuli that we used for the paired association task (adapted from Andreau and Funahashi, 2011).

Fig. 4. A. An example of pair selectivity observed in the sample-period activity of a prefrontal neuron (L06501). In this example, the greatest and second-greatest sample-period activities were observed when stimulus pair 9 was presented as the sample stimulus. B. Distribution of pair indices, whose values represent the strength of the pair selectivity of sample-period activity. The method used to calculate pair indices was described by Sakai and Miyashita (1991) (adapted from Andreau and Funahashi, 2011). 


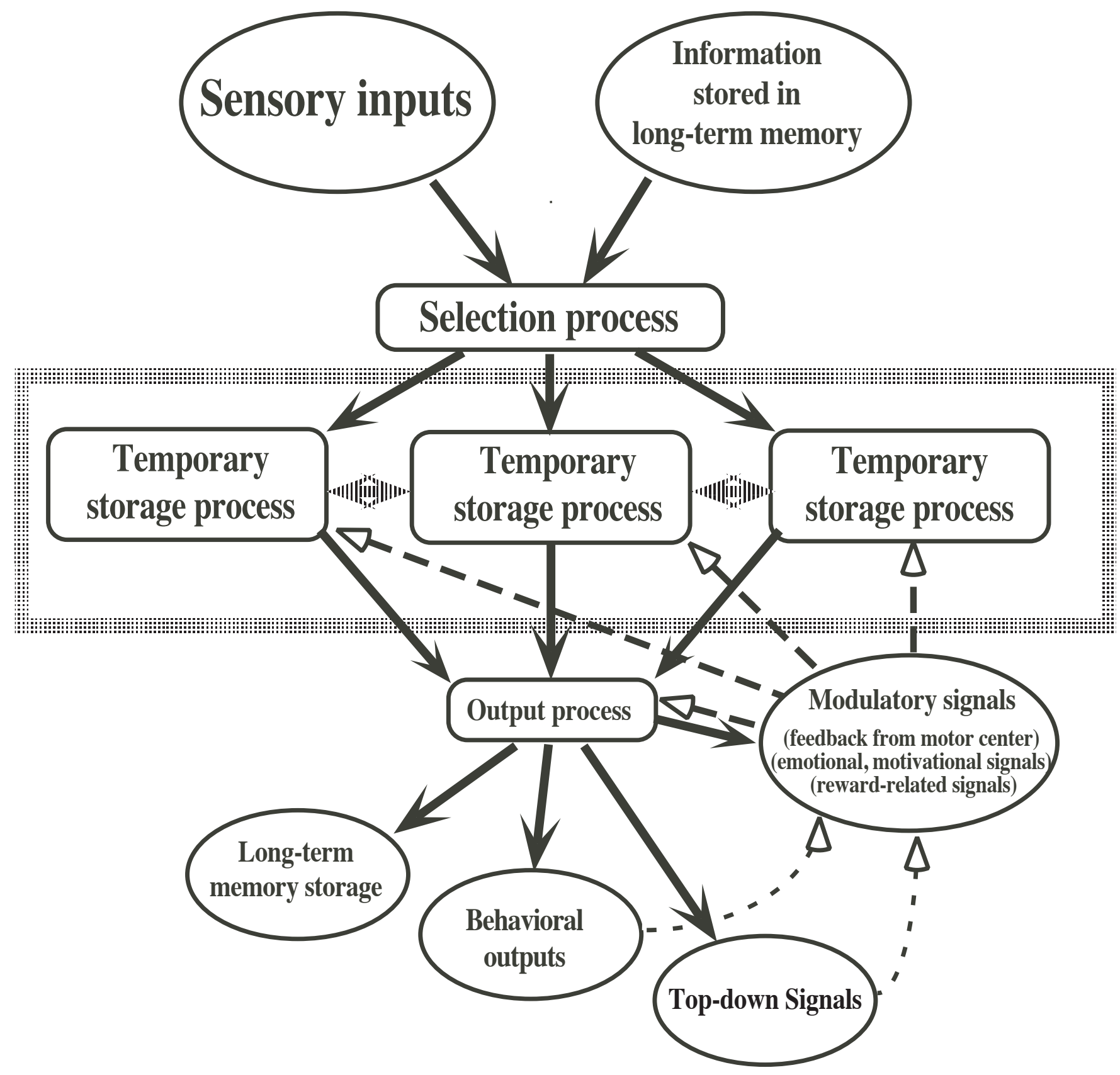

Fig. 1 


\section{monitoring}

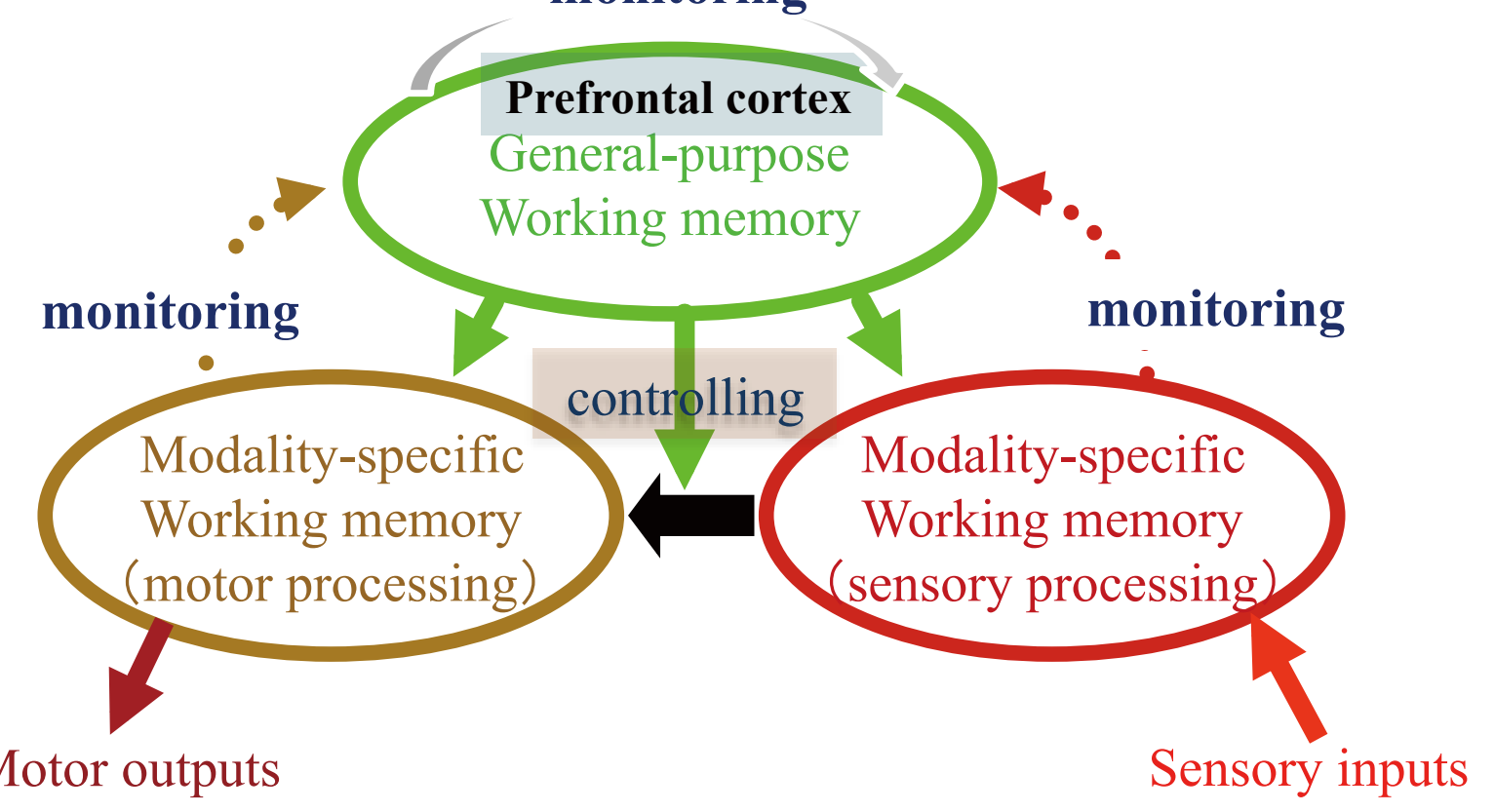

Fig. 2 


\section{A. Paired association task}



B. Visual stimuli used for the paired association task

Pair 1

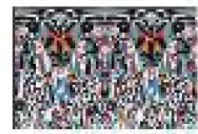

1

Pair 2

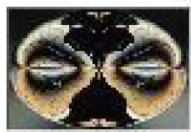

2

Pair 3



3

Pair 4

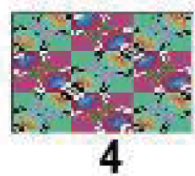

Pair 5



Pair 6

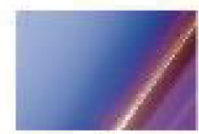

6

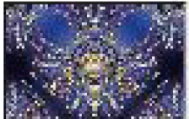

1

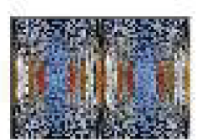

2'

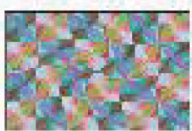

$3^{\prime}$

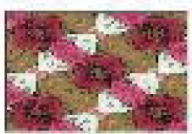

$4^{\prime}$

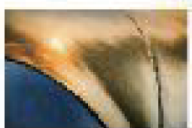

$5^{\prime}$

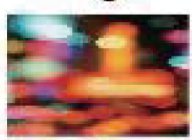

6'
Pair 7

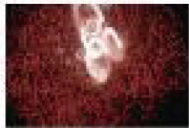

7

Pair 8

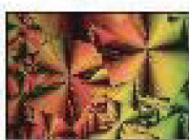

8

Pair 9

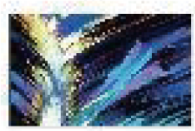

9



7

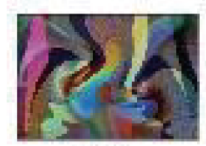

8'

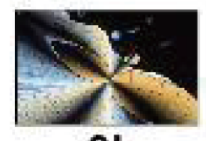

9'

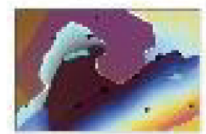

$10^{\prime}$

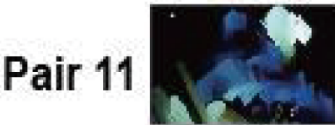

11

Pair 12

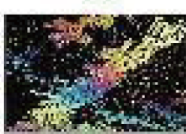

12

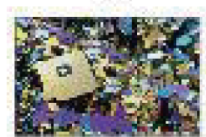

11'

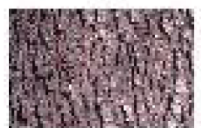

12 '

Fig. 3 


\section{A. Prefrontal pair-selective activity}

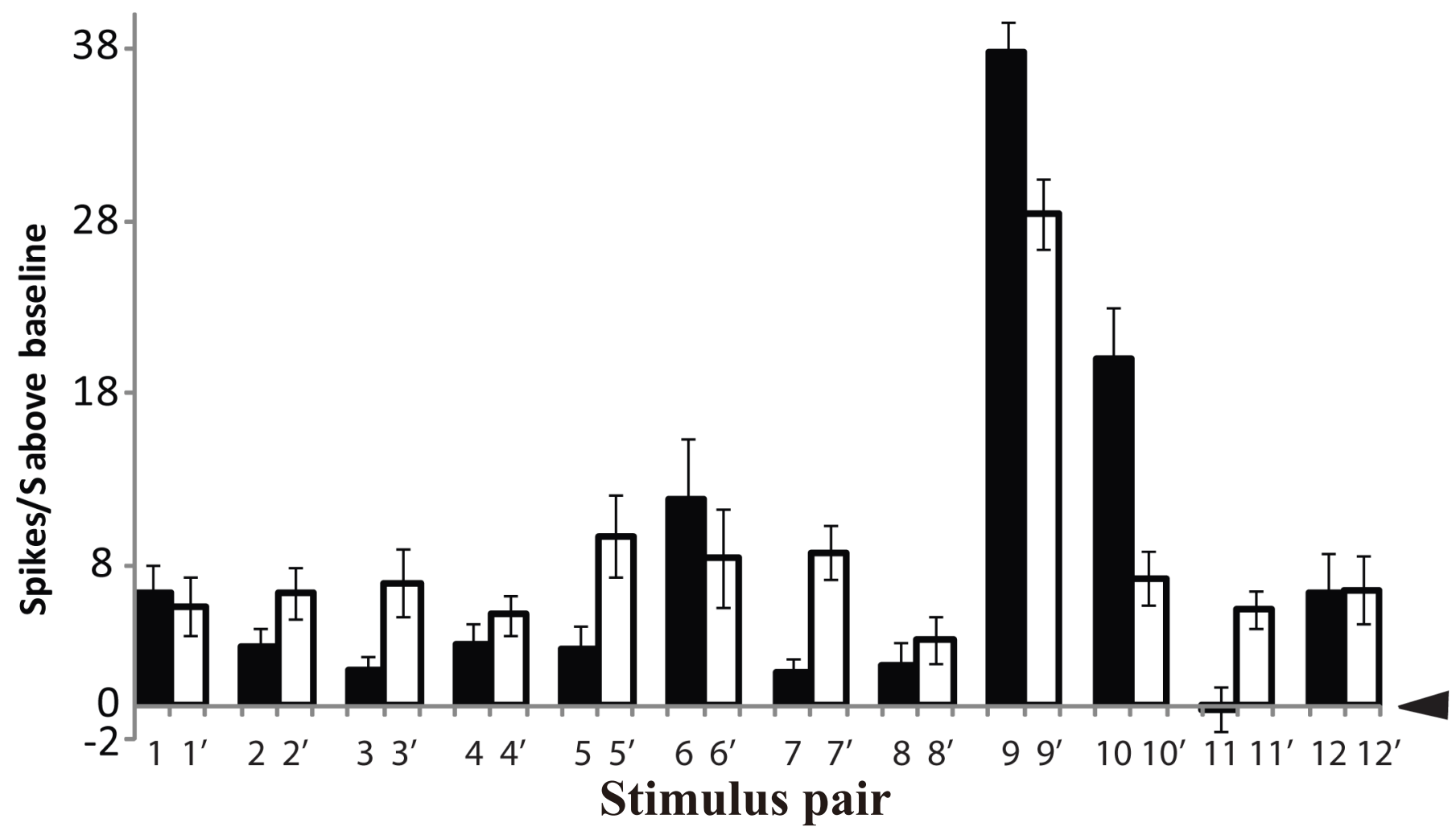

\section{B. Distribution of pair indices}

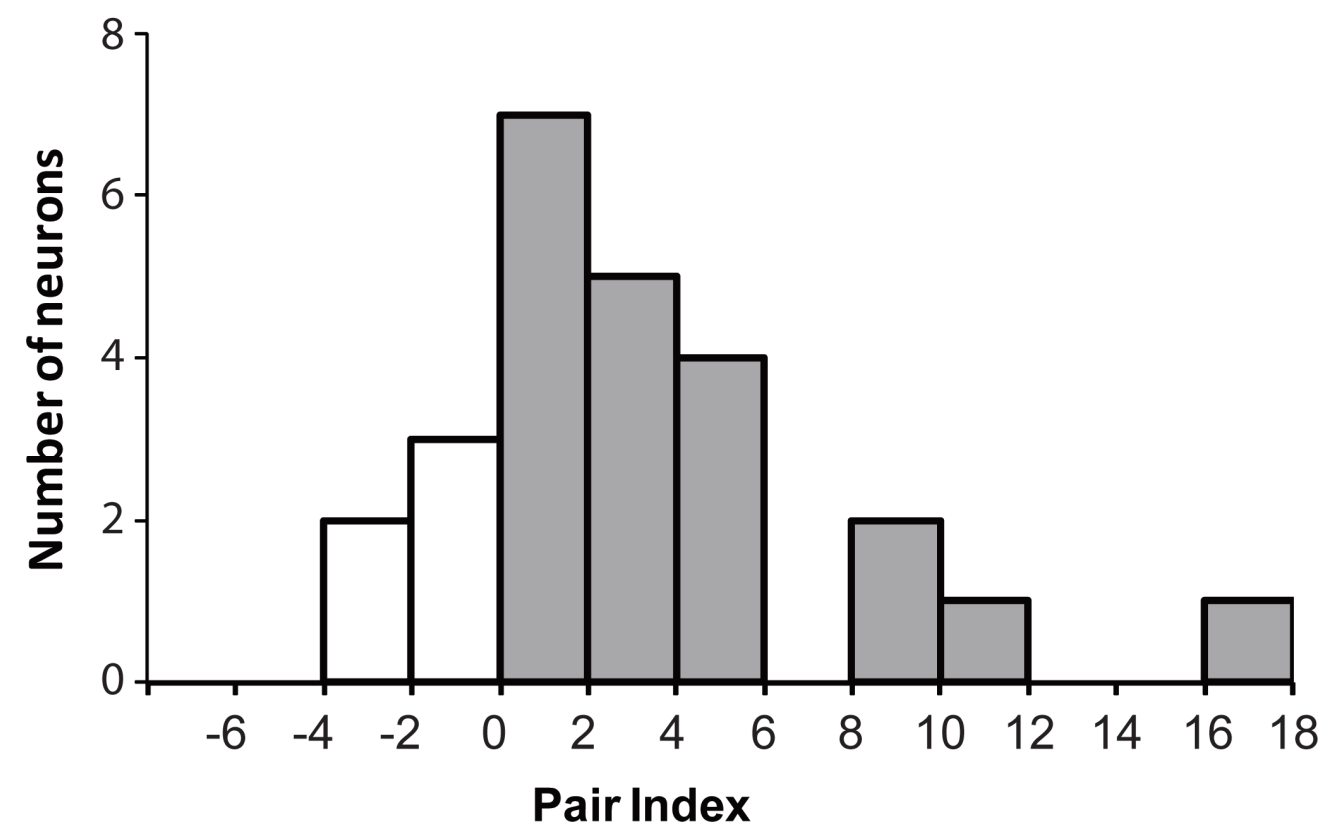

Fig. 4 